\title{
Human cloning: Safety is the issue
}

To the editor-A unique event occurred in the history of the reproduction of mammals when an adult lamb's nucleus was transferred into an enucleated ovum and led to the birth of the cloned lamb 'Dolly' public outcry from politicians and laity was immediate and almost instinctive: the use of adult nuclei for cloning humans should be banned (World Health Organization press release WHO/20, 11 March 1997; United Nations Educational, Scientific and Cultural Organization press release No. 9729, 28 February 1997; refs. 2,3). To many in the scientific community, it was repugnant to ban this fascinating area of research, especially as cloning could be foreseen to have beneficial consequences. They might have been more persuaded by arguments related to questions of the safety of the procedure rather than those related to the ethics, which appear to be vague and ill-formulated. Not prominently considered in the debate was the key issue of accumulated somatic mutations in the adult nucleus used for transfer possibly leading to premature aging, neoplasms and immunological disease. In an adult diploid nucleus more than $20-30 \%$ of enzyme loci are polymorphic ${ }^{4}$. It is probable that a similar extent of heterozygosity is found at the other 100,000 or more expressed loci of an adult nucleus. Many of these variant alleles may relate to disease but have no impact on phenotype because of the effects of the corresponding wild-type allele. Somatic mutation which occurs throughout each individual's lifetime can cause 'loss of heterozygosity' predisposing to the age-related development of cancers and immunological disease. The gene mutational rate can vary from 1 mutation in 100,000 cell divisions (meiosis or mitosis) to 1 in 10 depending on the stability of the allele in question ${ }^{5}$, and such mutations can accumulate in the nuclei of both somatic and germline cells.

In sexual reproduction, the zygote receives a random assortment of alleles from each parent, and previous somatic mutations of one parent are paired with alleles of the other, thus diluting the load of somatic mutations from each parent. In contrast, when a zygote receives its entire genetic complement from a nucleus derived from an adult tissue, further accumulation of somatic mutations during development may lead to increased risks of genetic abnormalities, especially when previously inactive genes become de-repressed in differentiating embryonic tissues. It would be outrageous to handicap a child from birth with a multitude of somatic mutations from the adult nucleus used for transfer, particularly as there is no possibility of informed consent for the experiment. This 'Catch 22 ' situation may very well forever block the use of cloning human organisms (but not organs) as the extent of expression of these mutant alleles after introduction into a human zygote will not be known until it is tried-and it is clearly unethical to try the experiment if there is a risk of harming the newborn child.

D.J. Galton, A. Kay \& J.S. Cavanna

Department of Human Metabolism and Genetics

St. Bartholomew's Hospital

London EC1A.7BE, UK

email d.j.galton@mds.qmw.ac.uk

1. Wilmut, I. et.al. Viable offspring derived from fetal and adult mammalian cells. Nature $385,810-813$ (1997).

2. The European parliament resolution on cloning. (Motion dated 11 March 1997, passed 13 March 1997, Brussels).

3. US Senate bills on cloning under fire from researchers. Nature 391, 623 (1998).

4. Hartl, D.L., Clark, A.G. in Principles of Population Genetics 2nd. edn. (Sinauer Associates, Massachusetts, 1989).

5. Vogel, F. in Principles and Practice of Medical Genetics 2nd. edn. Vol. 1(eds. Emery, A.H. \& Rimoin, D.L.) pp. 53-76 (Churchill Livingstone, Edinburgh, 1990).

\section{Cross-species infection: No news is good news?}

To the editor-Heneine and coworkers recently described occupational infections of four humans with simian foamy virus (SFV) and reported that none of three infected individuals transmitted SFV to their spouses and there has been no evidence of any diseases that might be related to SFV infection ${ }^{1}$. Although encouraging, these negative results should not be taken to suggest that SFV is a benign infection in humans and that as such we can proceed with animal organ transplantation to humans (xenotransplantation) using primates as organ donors.

Retroviruses by nature are insidious in their pathogenicity and can induce a wide spectrum of cancers. We have documented high rates of infection with SFV in captive baboons and have further demonstrated species-specific differences among SFVs in African primates indicating a long co-evolution of SFV and its respective host ${ }^{2}$. That a variety of viral and host factors influence retroviral pathogenesis has been clearly illustrated for primate immunodeficiency viruses $^{3}$. By analogy, SFVs may vary in a host-specific manner in their pathogenic potential. The fact that baboon SFVs were responsible for three of the four infections reported by Heneine et al. is surprising as macaques are more commonly bred in captivity and hence more likely to transmit infections to humans. Even subtle strainspecific differences may thus profoundly influence cross-species transmission and possibly disease which means that some SFVs may be more virulent than others.

Although SFV has not been transmitted by intimate contact in these cases, the persistence of SFV for more than 20 years in the peripheral blood of infected humans raises doubts that we can adequately protect the blood supply from viral contamination. Passage of virus by blood transfusion could dramatically enhance the pathogenicity and the potential dissemination of SFV through the blood supply. This type of passage might also provide an adaptive advantage, perhaps altering its virulence. We also know that SFV is very cytopathogenic in both simian and human cells in tissues culture-an important factor in assessing the potential for a virus to induce disease. Moreover, HTLV (human $\mathrm{T}$ cell lymphotropic virus) and a related retrovirus (STLV) found in baboons are weakly oncogenic, causing leukemias in less $4 \%$ of infected individuals after many decades of latency ${ }^{4}$. Baboons and other primates harbor several known pathogenic retroviruses and likely harbor several yet to be identified sources for human infections making baboons a risky proposition for xenografts. Thus although the lack of demonstrable disease in SFV infected persons is a good sign, we should resist the temptation to draw any conclusions regarding public health risks.

We also recently reported on the detection of SFV in several tissues taken at autopsy from two human recipients who died after receiving baboon livers ${ }^{5}$. Even if SFV was found to be relatively benign in humans, other resident viruses represent a substantial risk and this should steer us away from the use of non-human primates 